\title{
Avaliação do acesso de gestantes à atenção odontológica realizada pelo grupo PET-Saúde da Universidade Estadual De Londrina-PR
}

Jairo Augusto Marques de Carvalho*, Lucimar Aparecida Britto Codato ${ }^{* *}$, Octávio Henrique Carmona***, Ricardo Capello Papi***, Rodrigo Eik Sahyun***, Djamedes Maria Garrido*,Evelin Massae Ogatta Muraguchi****, Maura Sassahara Higasi****

* Preceptor do PET-Saúde da Família da UEL

** Tutora do PET-Saúde da Família da UEL

*** Alunos do PET-Saúde da UEL

**** Coordenadora do PRÓ-Saúde da UEL

$* * * * * \quad$ Coordenadora do Pet-Saúde da UEL

\section{RESUMO}

O grupo PET-Saúde da Universidade Estadual de Londrina que atua na Unidade Básica de Saúde Cambé II do município de Cambé-PR realizou pesquisa no período de maio a setembro de 2013 para verificar o acesso das gestantes que realizavam o prénatal na UBS ao programa odontológico e também para verificar o conhecimento, comprometimento e o referenciamento das gestantes para atenção odontológica pela equipe de saúde. A coleta de dados foi realizada por meio de questionários estruturados. Foram entrevistadas 26 gestantes de um total de 51 que realizavam pré-natal na UBS e 29 funcionários da UBS. Verificouse que $69,23 \%$ das gestantes conheciam o programa odontológico para gestantes; destas $77,77 \%$ receberam orientações na primeira consulta do pré-natal e agendaram a primeira consulta ao dentista. 94.44\% das gestantes receberam informações de forma verbal, por meio do médico obstetra, equipes de enfermagem e odontologia. Nenhuma agente comunitária de saúde transmitiu estas informações às gestantes. Quanto aos funcionários, $100 \%$ dos entrevistados afir- maram conhecer o programa odontológico para gestantes e ter conhecimento de que a equipe do PSF deve orientar as gestantes as gestantes para a atenção odontológica. Concluiu-se que a criação de instrumentos ou de estratégias padronizadas para a disseminação da informação, normatização e envolvimento de toda a equipe pode melhorar a logística e a adesão ao programa.

Descritores: Formação de Recursos Humanos; Sistema Único de Saúde; Saúde Bucal; Odontologia.

\section{INTRODUÇÃ̃O}

O Projeto PET-Saúde da Família da Universidade Estadual de Londrina 20122014 (PET-SF UEL) é formado por nove cursos de graduação da UEL (Educação Física, Enfermagem, Farmácia, Fisioterapia, Medicina, Medicina veterinária, Psicologia, Odontologia, Serviço Social). Atua em um projeto único de consolidação da Rede Cegonha, envolvendo 37 Unidades Básicas de Saúde de três municípios (Cambé, Londrina e Ibiporã), integrado à $17^{\mathrm{a}}$ 
Avaliação do acesso de gestantes à atenção odontológica realizada pelo Grupo PET-Saúde da Universidade Estadual De Londrina-PR

Regional de Saúde do Paraná e ao Programa de Qualificação da Atenção Primária à Saúde - APSUS da Secretaria Estadual de Saúde do Paraná.

O projeto foi criado de modo colaborativo com representantes de todos os segmentos envolvidos, além de representantes da Pró-Reitoria de Graduação da UEL e de representantes dos Conselhos Municipais de Saúde. Inicialmente planejado para 12 grupos. Porém, quando houve a aprovação de 5 grupos, vários preceptores e estudantes se motivaram a continuar no Projeto como voluntários, o que permitiu que mantivéssemos a totalidade das UBS iniciais. Temos hoje 5 tutores, 60 preceptores (30 voluntários) e 160 estudantes (100 voluntários), todos focados no objetivo geral que é a redução da mortalidade materno-infantil, por meio da consolidação das Redes de Atenção à Saúde (Mãe Cegonha). As ações do Projeto têm possibilitado avanços na integração com as três Secretarias de Saúde, a formação de um profissional segundo as DCN e também momentos de Educação Continuada e Permanente dos sujeitos envolvidos.

No primeiro semestre do projeto foram realizadas a territorialização, a elaboração de mapas epidemiológicos, em paralelo ao embasamento teórico-prático dos protocolos existentes. No inicio do segundo semestre foram construídos e desenvolvidos projetos de intervenção baseados nas fragilidades identificadas durante a territorialização e também por meio de relatos de demandas realizados pelos profissionais da rede de serviços.

O grupo PET-Saúde da Universidade Estadual de Londrina que atua na Unidade Básica de Saúde (UBS) Cambé II, em trabalho conjunto com os profissionais da UBS, verificou a necessidade de se identifi- car a realidade do acesso de gestantes à atenção odontológica.

Sabe-se que o pré-natal é um período ímpar na vida da gestante. Vários estudos apontam a abertura da gestante para absorver novas informações e hábitos, que podem resultar em melhorias do seu próprio cuidado e também no do bebê. . $^{3,4,5,8,10}$

Há necessidade de programas de atenção odontológica direcionados a gestantes por se tratar de um grupo estratégico devido às características psicossociais inerentes ao processo reprodutivo e, também em razão do papel que as mães desenvolvem na promoção de uma melhor saúde bucal de seus filhos. ${ }^{2,7,10,11,12}$

Em 2005, o município de CambéPR implantou as Equipes de Saúde Bucal (ESB). Nesse momento, foram realizadas reuniões com os funcionários da UBS para esclarecimentos em relação à mudança do modelo de atenção que, até então, priorizava o atendimento de as crianças até 12 anos de idade.

Com a inserção das ESB em todas as UBS, o município em estudo ampliou o cuidado para todas as faixas etárias, com inclusão de grupos de usuários com características específicas, como exemplo as gestantes.

O protocolo de implantação das ESB orienta a abordagem das gestantes que realizam o pré-natal na UBS, de preferência no primeiro trimestre da gravidez, para que a equipe possa trabalhar com a futura mãe em ações educativas, preventivas e curativas. $^{9}$

O grupo PET-Saúde da Família que atua na UBS- Cambé II, em trabalho voltado para o diagnóstico local, constatou falta de uniformidade na abordagem das gestantes para agendamento na consulta odontológica, considerada pela equipe uma fragilidade para o cuidado da gestante por meio 
do Programa Odontológico para as Gestantes (POG), que está em funcionamento na UBS Cambé II desde setembro de 2005.

Na UBS em estudo, os funcionários sabem que existe o programa odontológico para as gestantes, porém não existe uma sistematização no encaminhamento das gestantes para o atendimento odontológico. A equipe de saúde relatou as seguintes dúvidas: Quem é responsável pelo encaminhamento da gestante para a avaliação odontológica? Como pode ser feito o agendamento? Em qual momento a gestante deve ser abordada e encaminhada para a avaliação com o dentista da UBS? As gestantes conhecem o programa odontológico que lhes é oferecido pela UBS? Estão sendo abordadas e encaminhadas? Sabem a importância deste atendimento? Conhecem a bebê clínica?

O grupo PET-SF constatou que o acesso das gestantes ao programa odontológico estava deficiente quando analisou o fluxo das mesmas para agendamento para no o atendimento no POG.

É fato que o acesso das gestantes a programas odontológicos é influenciado por vários fatores como: mitos, medos, falhas na comunicação, falta de informação bem como falta de capacitação do próprio dentista para desenvolvimento do atendimento. ${ }^{1,6}$

A falta de informação pode gerar insegurança nas gestantes e também nos profissionais. ${ }^{10}$ As gestantes podem e devem ser esclarecidas e motivadas, o que justifica a adoção de programas voltados para o cuidado da gestante, tanto no âmbito dos serviços públicos como também dos privados. $5,11,12$

Para melhoria da atenção odontológica à gestante, são necessários investimentos em educação permanente e continu- ada dos profissionais envolvidos na prestação da atenção. Incluem investimentos públicos que possibilitem maior e melhor acesso aos serviços, somado à constante avaliação da estrutura, do processo e dos resultados dos serviços de saúde. ${ }^{2}$ Neste contexto, o presente estudo teve como objetivos obter informações sobre como tem se dado encaminhamento das gestantes ao Programa Odontológico para Gestantes (POG) oferecido pela UBS em estudo.

\section{METODOLOGIA}

A coleta de dados aconteceu no período de maio a setembro de 2013, como atividade do Programa PET Saúde/ UEL 2013, por meio de aplicação de questionário direcionado às gestantes para avaliar: o conhecimento desse grupo em relação à existência e ao funcionamento do POG e como tem se dado esse processo durante o período gestacional.

A pesquisa também aplicou questionário para os funcionários da UBS para analisar como tem se dado o referenciamento das gestantes para a atenção odontológica.

Foram entrevistadas 26 de um universo de 51 gestantes que realizavam prénatal na UBS Cambé II e a 29 dos 36 funcionários lotados na UBS que livremente aceitaram participar da pesquisa.

\section{RESULTADOS E DISCUSSÃO}

Verificou-se que $69,23 \%$ das mulheres pesquisadas conheciam $\mathrm{o}$ programa odontológico para gestantes; $77,77 \%$ responderam ter recebido orientações na primeira consulta do prénatal, de forma verbal, por meio da enfermeira, auxiliares de enfermagem e do obstetra. $16,66 \%$ receberam a informação por meio da equipe de saúde bucal. 
Avaliação do acesso de gestantes à atenção odontológica realizada pelo Grupo PET-Saúde da Universidade Estadual De Londrina-PR

Verificou-se que nenhum Agente Comunitário de Saúde (ACS) encaminou gestantes para o POG. $77,77 \%$ agendaram a primeira consulta ao dentista; destas $78,57 \%$ compareceram à primeira consulta; $81,81 \%$ estão realizando o tratamento proposto, $100 \%$ consideram a saúde bucal importante para a saúde geral e pretendem trazer seus filhos para acompanhamento odontológico precocemente, antes dos seis meses de idade.

Quanto aos funcionários entrevistados (11 auxiliares de enfermagem, 08 ACS, 03 auxiliares administrativos, 01 auxiliar de serviços gerais, 03 médicos e 03 enfermeiras) verificou-se que $100 \%$ dos funcionários afirmaram conhecer $\mathrm{o}$ programa odontológico para gestantes da UBS e afirmaram ter conhecimento de que a equipe do PSF deve orientar as gestantes que fizeram o pré-natal ao agendamento odontológico; $93 \%$ responderam que orientam as gestantes a agendarem consulta odontológico de forma verbal; $100 \%$ acham importante padronizar a entrega de um folder informativo sobre o programa. Quando questionados sobre o melhor momento para realização da abordagem da gestante, $17,24 \%$ responderam que seria na primeira consulta do pré-natal com a enfermagem, $20.68 \%$ acham que deveria ser feito pelo ACS, $6.89 \%$ com a enfermagem e obstetra, $10,34 \%$ com a enfermagem e ACS e $44,82 \%$ com a enfermagem, obstetra e ACS. Com relação à responsabilidade do encaminhamento das gestantes para o agendamento no programa odontológico, 93\% responderam que toda a equipe deve deve encaminhar as gestantes.

A análise dos resultados revelou conflito de informações. 93\% dos funcionários responderam que orientam as gestantes quanto ao agendamento da consulta odontológica, por outro lado apenas $69,23 \%$ das gestantes conheciam o programa odontológico para gestantes. Uma possível explicação seria a existência de falhas na comunicação.

Outro aspecto evidenciado pelo estudo foi a falta de consenso entre os funcionários quanto ao melhor momento para realização da abordagem da gestante. Chamou a atenção a atenção a ausência de ACS no encaminhamento de gestantes , evidenciando a necessidade de avanços no envolvimento de todos os profissionais na abordagem e no cuidado integral da gestante.

\section{CONSIDERAÇÕES FINAIS}

O estudo evidenciou a necessidade de educação permanente de toda equipe no cuidado da gestante, pois há rotatividade de funcionários da UBS em função de mudanças de local de trabalho, exoneração, aprovação em outro concurso, ingresso de novos funcionários novos.

A falta de uniformidade na abordagem das gestantes para agendamento na consulta odontológica aponta a necessidade de estabelecimento de fluxo e envolvimento de todos os profissionais no encaminhamento das gestantes ao programa. Há necessidade de criação de instrumentos ou de estratégias padronizadas para a disseminação da informação, criação de protocolos que contribuam avanços no processo de trabalho e com o envolvimento de toda a equipe pode melhorar a logística e a adesão ao programa.

O grupo PET-Saúde apresentou os resultados do estudo para a equipe de saúde local. Como estratégia de intervenção, elaborou um folder explicativo sobre o cuidado odontológico no período gestacional e sobre o fluxo de atendimento na UBS local. 
Avaliação do acesso de gestantes à atenção odontológica realizada pelo Grupo PET-Saúde da Universidade Estadual De Londrina-PR

Sugeriu que o folder fosse utilizado por toda a equipe.

Importante destacar que a participação do grupo Pet-saúde nessa pesquisa foi muito positiva para o os alunos porque possibilitou aprendizado em situações reais e também para os profissionais de saúde porque possibilitou o conhecimento da realidade e a proposição de estratégias de ação para o enfrentamento das demandas.

\section{REFERÊNCIAS}

1. Badeia M. Sistemas de atenção em odontologia. Vias de acesso. Rev. Paul.Odontol1991 set./out.; 13(5): 2-14.

2. Codato LAB. Pré-natal odontológico e saúde bucal: percepções e representações de gestantes. 2005. 131 f. Dissertação (Mestrado em Saúde Coletiva) Universidade Estadual de Londrina, Londrina, 2005.

3. Corsetti LO, et al. Avaliação do atendimento odontológico para gestantes nos serviços públicos de Porto Alegre/RS, durante o pré-natal. ABOPREV 1998 nov; 1(1): 9-5.

4. Costa ICC, et al. A gestante como agente multiplicador de saúde.Rev. Pós Grad1998 5(2):87-92.

5. Maeda FHI, et al. A visão das gestantes quanto àscondutas odontológicas na cidade de Franca. UFES Rev. Odontol 2001 jul./dez; 3(2):8-14.

6. Moimaz SAS, Rocha NB, Saliba O, Garbin, CAS. O acesso de gestantes ao tratamento odontológico. Revista de Odontologia da Universidade Cidade de São Paulo 2007 jan-abr; 19(1): 3945 acessado em 30-09-20013 http://www.cidadesp.edu.br/old/revista _odontologia/pdf $/ 3$ janeiro_abril 2007 lo_acesso gestantes.pdf
7. Moreira PV, et al. Uma atuação mulltidisciplinar relacionada à promoção de saúde oral materno-infantil.Pesq. Bras. Clin. Integr 2004 Set./Dez; 4(3): 25964.

8. Moura LFD, et al. Apresentação do programa preventivopara gestantes e bebês. JBP 2001 jan./fev.4(7): 10-4.

9. Prefeitura Municipal de Cambé. Secretaria Municipal de Saúde - Departamento de Atenção Integral à Saúde Divisão de Serviços Odontológicos. Protocolo de Atenção Odontológica do Município de Cambé - Pr. / Divisão de Serviços Odontológicos- Cambe- Paraná, 2006.

10. Rossel FL. Prevalência de fatores clínicos do riscode cárie em gestantes. Araraquara, 1998. Tese (Doutorado)- Faculdade de Odontologia da Universidade Estadual Paulista "Júlio de Mesquita Filho".

11. Sartorio ML, Machado WAS. A doença periodontal na gravidez. Rev. Bras. Odontol2001 Set/Out; 58(5): 306-8.

12. Scavuzzi AIF et al. Estudo da prevalência de doença periodontal em gestantes brasileiras residentes em Salvador - BA. ROBRAC 1999 18(25): 405.

\section{ABSTRACT \\ Pregnant's dental care acess evaluation conducted by PET-Health group at the State University of Londrina}

Evaluation of pregnant women access to dental services provided by the Londrina State University/PR PET-Health group II Basic Health Unit carried out a research from May to September 2013 to verify the access of pregnant women who were receiving prenatal care at that UBS to the Dental Program and also to verify the 
knowledge, commitment and the referrals of these women to dental care by the health team. Data the PET-Health group from the Londrina State University that provides services at the Cambé were collected through structured questionnaires. Twentysix (26) pregnant women out of the 51 who were receiving prenatal care at the UBS were interviewed as well as 36 UBS employees. Results showed that $69,23 \%$ of the pregnant women knew about the Dental Program; $77,77 \%$ of them received orientations during the first prenatal consultation and scheduled their appointment with the dentist; $78,57 \%$ went to the first consultation; $81,81 \%$ are currently under dental treatment; $100 \%$ consider oral health important to general health; $100 \%$ intend to bring their kids to early dental follow-ups; $94,44 \%$ of the pregnant women received information verbally, by their obstetrician, nursing and dentistry teams. None of the health agents gave this information to the expecting mothers. As for the employees, $100 \%$ of the interviewees stated that they learned about the dental program for pregnant women and that the PSF team should provide guidance to them towards the importance of dental care. This study concluded that the development of standardized instruments or strategies to disseminate information, the establishment of norms and the involvement of the whole team can improve the logistics and increase participation in the program.

Descriptors: Human Resources Formation ; Unified Health System ; Oral Health; Dentistry 